\title{
Analyzing jokes with the Intersecting Circles Model of humorous communication
}

\author{
Francisco Yus \\ University of Alicante
}

\section{Introduction}

In previous research, I have claimed that relevance theory (Sperber and Wilson 1995 ) is a valid cognitive pragmatics framework to explain how humorous effects are generated, both from the processing of jokes (Yus 1997, 2003, 2008, 2012a, $2012 \mathrm{~b}$ ) and from the processing of longer discourses such as stand-up comedy monologues (Yus 2002, 2004, 2005). This paper aims to show how the speaker's humorous intention is devised and how humorous effects are generated in the audience by analyzing 1000 randomly collected jokes and checking how they fit into any of the seven types of jokes that are predicted in the Intersecting Circles Model of humorous communication (Yus forthcoming). The paper is organized as follows: First, I provide a brief comment on the cognitive foundation of relevance theory and the inferential steps that hearers go through in the interpretation of utterances according to this theory. Second, I comment on how these steps can be manipulated for the sake of humor. Next, comments on a previous classification of jokes are provided. Finally, the Intersecting Circles Model of humorous communication (henceforth ICM) is outlined and the corpus of jokes is analyzed to check how they fit into the seven types of jokes that this Model comprises.

\section{Relevance theory and human cognition}

Research within relevance theory assumes that all cognitive activity of the human mind is relevance-oriented. Inputs for mental processing are attended to because we have developed a tendency to search for their optimal relevance. This is captured in the cognitive principle of relevance: "Human cognition tends to be geared to the maximization of relevance." According to Wilson and Sperber (2002: 251), "relevance is a potential property not only of utterances and other observable phenomena, but of thoughts, memories and conclusions of inferences." Concerning our thoughts, these are also graded by relevance, since some of them are more likely to be entertained than others in a particular situation. In general, any external stimulus or internal representation that accesses our cognitive system may be 
relevant to us at some time and we have an evolved ability to pick up potentially relevant stimuli. For an input to be relevant, it has to combine fruitfully with context to yield interesting outcomes ("positive cognitive effects" in relevancetheoretic terminology). Indeed, human cognition is constantly gathering information and combining it with contextual information to yield relevant conclusions. For this to happen, the potential interest of the input should not demand excessive processing effort in return. In this sense, if extra effort is demanded (for instance, when the interpretation is communicated as an implicature which requires a lot of contextualization), this effort should be compensated for with additional cognitive effects. Humans invariably tend to select the most accessible and effort-relieving interpretation by default unless this interpretation proves not to be relevant enough, for example when more context needs to be accessed in order to reach an adequate interpretation. Jokers often foresee their interlocutors' tendency to select interpretations in terms of accessibility and potential relevance and manipulate this tendency with alternative unpredicted interpretations (more on this below).

To summarize, four are the basic assumptions of relevance theory (Wilson 1994: 44). These will be exemplified with Mike's answer in (1) and the range of its possible interpretations in (2) below. As will be described in the next Sections, the same cognitive properties that make human comprehension possible, are also at work in the generation of humorous effects.

(1) John: Now, tell me, how's your girlfriend?

Mike: She's no longer my girlfriend.

John: Oh! I am really sorry... I really thought you got on very well with each other.

Mike: No! I mean... she is now my wife! We got married last month.

John: Oh! I see... Congratulations! I had no idea!

(2) a. She's not my girlfriend because my girlfriend has died

b. She's not my girlfriend because my girlfriend and I split up.

c. She's not my girlfriend because my girlfriend and I are now married.

The first theoretical assumption of relevance theory is that every utterance has a variety of possible interpretations, all compatible with the information that is linguistically encoded. In (1), Mike's answer has a number of possible interpretations (2a-c), all of which are compatible with this utterance and, on paper, possible in the context in which it is uttered ((2a-c) are "manifest" in relevancetheoretic terminology).

The second assumption is that not all these interpretations occur to the hearer simultaneously; some of them take more effort to think up. John chooses interpretation ( $2 b$ ) because it takes less effort to arrive at than interpretation (a) or (c). After all, "splitting up" is the most typical reason why a woman stops being a man's girlfriend. 
The third assumption is that hearers are equipped with a single, general cognitive criterion for evaluating the relevance of potential interpretations. Indeed, humans are fast at evaluating interpretations, to the extent that John does not even realize that there are alternative interpretations (2a) and (2c) when he selects (2b) as the intended one.

Finally, this criterion is powerful enough to exclude all but a single interpretation, so that having found an interpretation that fits the criterion, the hearer looks no further. In the example above, once John selects (2b) as the intended interpretation, he does not consider the possibility that there might be other competing interpretations such as (2a) and (2c).

\section{Comprehension according to relevance theory}

Relevance theory claims that comprehension involves (a little bit of) decoding and (a great deal of) inference. Each chunk of text is decoded by a specialized module of the brain and turned into (part of) the logical form of the utterance. This is the only context-free phase of utterance interpretation. The logical form is a grammatical string of words which is communicatively useless unless it is enriched pragmatically and turned into a relevant contextualized proposition. As shown in Figure 1, utterance interpretation involves a mutual parallel adjustment of (a) inferential operations to yield the explicit content of the utterance or explicature; (b) the retrieval of implicated premises and derivation of implicated conclusions or implicatures (if necessary); and (c) the access to as much contextual information as necessary to obtain (a) and (b).

INTERPRETATION AS MUTUAL PARALLEL ADJUSTMENT

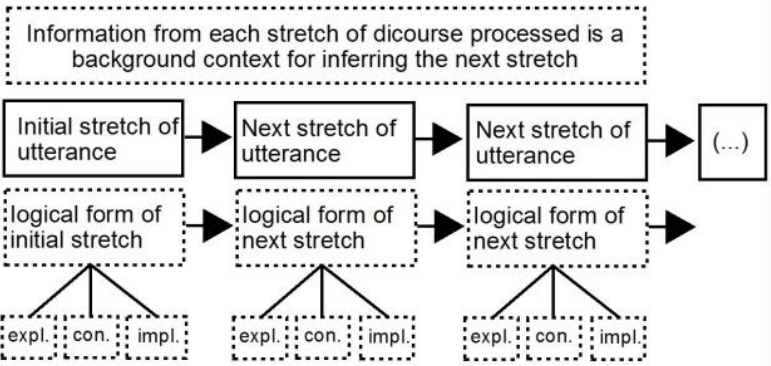

Figure 1. Interpretation as mutual parallel adjustment of explicature (expl.), implicature (impl.) and context (con.). 
The enrichment of the logical form to obtain its explicit interpretation (explicature) involves several possible inferential procedures such as reference assignment (especially for indexicals, adverbs, etc. as in (3a)), disambiguation (of polysemous words, as in (3b)), free enrichment of non-coded elements (3c); and adjustment of concepts, when the coded concept is altered to meet the expectations of relevance (leading to the inferential construction of an ad hoc concept that only resembles the coded one in some respect), either by turning it into a more specific or narrower concept, as in $(3 \mathrm{~d})$, or by turning it into a less specific or broader concept, as in (3e):

(3) a. I saw her there with him.

I saw [whom?] there [where?] with him [whom?].

b. I came across Thomas this morning. He was at a bank. [river bank? financial institution?].

c. I think Ann is better.

I think Ann is better [than whom?] [for what?].

d. John drinks too much.

John [drinks alcohol] too much.

e. I'll be ready in two minutes.

I'll be ready [in a while].

And the explicature, together with contextual information, is used as a premise for the derivation of implicatures (if intended). Contextual assumptions are needed both for the retrieval of implicated premises and for the derivation of implicated conclusions, as in the hypothetical (4b) and (4c) below:

(4) a. Mike: Are you going to next Saturday's party?

Ann: My parents are away this weekend.

b. Implicated premise: Ann has to look after her grand-mother when her parents are away.

c. Implicated conclusion: Ann won't be able to go to next Saturday's party.

During comprehension, hearers often make anticipatory hypotheses and can derive implications even before the explicit interpretation of the utterance has been fully processed. This has often been underlined as important for incongruityresolution in humor, although predictions are often made for "frame congruence" rather than for actual content (Dynel 2012a: 8). Similarly, hearers are often forced to backtracking when the subsequent stretch of discourse invalidates the assumptions brought to bear so far in the interpretation of the utterance. This is also typical of jokes fitting the incongruity-resolution pattern. In any case, as represented in Figure 1, interpreting an utterance is an incremental heuristic in which the assumptions obtained from the processing of previous chunks of text form a preliminary context for the interpretation of subsequent chunks (Dynel 
2012b: 158), an inferential procedure that may be exploited for the sake of humor (see below). As Ritchie (2004: 49) correctly stresses, "the oddity of incongruity does not involve a configuration of objects perceived all at the same time, but the temporal sequence of events or ideas creates the effect."

\section{Relevance theory, humor and joke classification}

Although humans cannot enter other people's minds, we are endowed with a mindreading ability that aids in metarepresenting other people's thoughts, together with predictions of context accessibility and mutuality of assumptions. Therefore, speakers can predict that certain interpretive steps will be taken, that one of the senses of a word is bound to be picked up because it offers a better balance of cognitive effects and processing effort, or that certain background assumptions will be retrieved from context for the derivation of implicatures. In a nutshell, speakers can somehow foresee the inferential steps of the interlocutor and act upon them as part of their humorous strategy. In fact, all the steps of interpretation commented upon in Section 3 above can be manipulated in jokes: the context-free extraction of the logical form of the utterance, the inferential enrichment of the coded words (reference assignment, disambiguation, free enrichment and concept adjustment) and the access to contextual assumptions needed for the retrieval of implicated premises and the derivation of implicated conclusions (cf. Yus, 2003, 2008).

Relevance theory also predicts that, upon interpreting the utterance, the hearer will make sense of a proper scenario for the joke in the form of schemas, frames, scripts, etc. (see below for my proposal of the unifying term make-sense frame); and this scenario may also be manipulated for the sake of humor. For instance, the incongruity-resolution pattern of humor fits this manipulation (which may also rely on a linguistic focus of incongruity, besides frames; see Suls 1972: 82, Ritchie 2009: 11).

The different areas of interpretation that can be manipulated as part of the intention to generate humorous effects constitute the basis for classifications of jokes made in previous research (cf. Yus 2003, 2008, 2012a, 2012b). Figure 2 shows one of the latest proposals. The chart starts with a differentiation between intentional humor and unintentional humor, the latter not covered by a pragmatics of humorous communication. Then, a distinction is made between the jokes which are integrated into the on-going conversation (and hence catch the interlocutor by surprise) and those which are not (canned jokes, typically preceded by markers such as "have you heard the one...?"). In both cases, jokes are then divided into those whose humorous effects lie in the manipulation of the inferential steps leading to an interpretation of the joke, and those which simply strengthen (or 
occasionally contradict) social and cultural stereotypes regarding national features, sex roles, professions, celebrities, connoted places, etc.

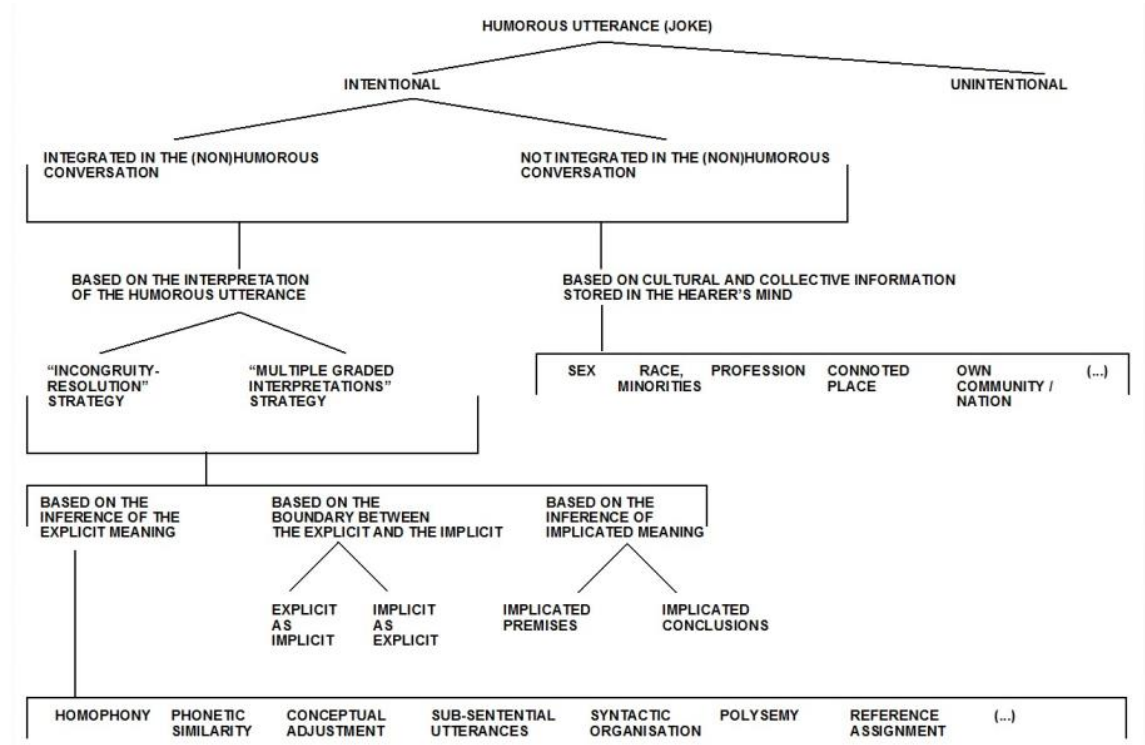

Figure 2. A relevance-theoretic classification of jokes (Yus 2008, 2012a).

For the former (utterance-centered humor), two basic strategies are cited: the incongruity-resolution pattern, and what has been labeled "multiple graded interpretations strategy" (Yus 2003: 1309), which also covers the "incongruityresolution" model (Suls 1972), the "forced-reinterpretation model" (Ritchie 2004, 2006), the "marked informativeness criterion" (Giora 1991) and the "garden-path mechanism" (Dynel 2012b). According to this strategy, the speaker plays with multiple interpretations of the joke which are graded according to their accessibility. Finally, the next layer in Figure 2 covers all the sub-strategies centered upon the aforementioned steps leading to an interpretation of the joke (reference assignment, disambiguation...).

\section{The Intersecting Circles Model (ICM) of humorous communication}

Although I never meant the types of jokes represented in Figure 2 to be mutually exclusive (especially the utterance-centered versus the cultural stereotype-connoted 
division), this classification has been criticized on these grounds (cf. Dynel 2010, 2012b; Biegajło 2012). However, I do consider the role of different sources of humor and their combination in single instances of jokes worth pursuing. This is why in Yus (forthcoming) a brand new proposal of classification is suggested in which it is claimed that all jokes involve the speaker's prediction (and/or manipulation) of certain interpretive steps and context accessibility within three areas generically labeled Circles: (a) the make-sense frame (the overall interpretation of the scenario depicted in the joke), (b) the cultural frame (encyclopedic information concerning cultural and social stereotypes on religions, sex roles, connoted places, celebrities, etc.), and (c) utterance interpretation (the inferential steps taken to turn the words of the joke into a meaningful interpretation). ${ }^{2}$ The combination of these circles make up the ICM of humorous communication comprising seven types of jokes (Figure 3).

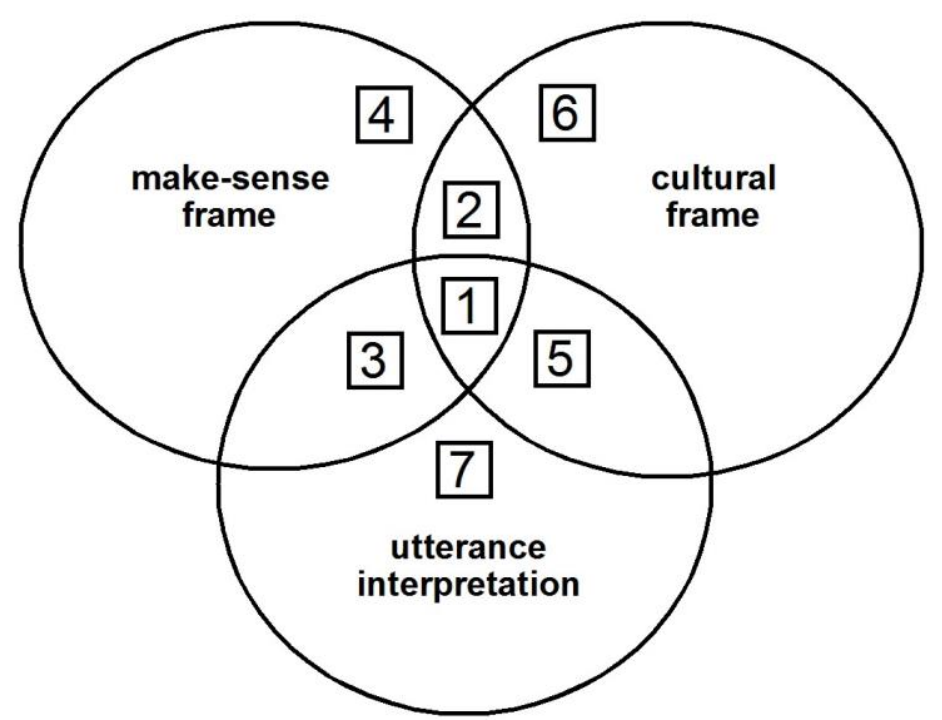

Figure 3. The Intersecting Circles Model (ICM) of humorous communication.

Before showing the outcome of the analysis of a large corpus of jokes, the three circles will be briefly described in the next Sections.

\subsection{Circle 1: Make-sense frame}


Inferring the intended interpretation of the utterance involves the retrieval of information that is stored as accessible chunks of encyclopedic information (specifically stored as "I conceptualize $\mathrm{X}$ as $p$ " or as a more factual "I believe that $p$ "). This information is often constructed on the fly and almost unconsciously in order to make sense of the intended "scenario" for the comprehension of utterances. But the terminology used for describing this kind of mental storage is not uniform across authors and research schools.

Firstly, "frame" is a widely used term for this kind of commonsense information about the world and repeated situations, capturing the 'typical' features of the world, stereotyped situations, etc. Secondly, the term "schema" is often suggested for this kind of default general information. These are basic chunks of information that allow us to engage in daily experiences and classify them as prototypical instances. Finally, "script" is also commonly used in the analysis of this kind of information, but it refers more to sequences of actions. When the script is mentally activated, we expect certain events to take place in a certain order.

The scopes of these terms overlap enormously and many analysts treat them as interchangeable (cf. Tannen 1993). For example, Scollon and Scollon (2001: 67) seem to treat schemas and scripts as similar concepts. Bednarek (2005: 688) comments that these competing terms usually differ only in emphasis and cannot be easily distinguished. Therefore, I propose to group all of these terms under the generic label of make-sense frame. This term comprises three types of chunks of information that we invariably retrieve by default during comprehension:

(a) Word-associated schemas. We tend to attach a number of cognitive "encyclopedic features" (as I prefer to call them) to the referents of the words that we use in our daily interactions. These overlap to a greater or lesser extent with other people's schemas of the same word, but differences arise both in personal and cultural backgrounds. In the case of metaphors, some inference is typically devoted to adjusting these conceptual features so that they can be effectively mapped onto target domains.

(b) Sequence-associated scripts, such as "coffee-shop," that contain a number of prototypical, taken-for-granted actions such as finding a seat, determining one's order, placing one's order with the waiter or waitress, receiving one's food and paying the bill at the cashier's (Scollon and Scollon 2001: 67).

(c) Situation-associated frames, based on an accumulation of words whose conceptual referents suggest a prototypical situation such as "being at the doctor's" or "buying food at the supermarket." Instead of a list of predicted actions, as in (b) above, situation-associated frames are accumulations of concepts regarding a prototypical situation.

Needless to say, these make-sense frames are often exploited as part of a humorous intention, normally because the frame brought to bear so far in the interpretation of a stretch of the joke is inconsistent with the subsequent stretch and 
has to be changed (in Coulson's 2001 terminology, there is often a frame shift during the interpretation of the joke). Indeed, the role of make-sense frames has been underlined in several theories of humor with variations in the semanticspragmatics interface. It is found in Koestler's (1964) bisociation theory, according to which humor involves two self-consistent but totally incompatible frames of reference. It is also central in the Semantic Script Theory of Humor (Raskin 1985) and its continuation in the General Theory of Verbal Humor (Attardo and Raskin 1991), whose central assumption is that information in the joke can be represented by abstract knowledge structures called "scripts" which often clash. Scripts are also present in informativeness approaches to jokes (cf. Attardo 2011: 141), according to which the hearer's tendency to remain within one script clashes with a subsequent script. Finally, frames are also essential in Wenzel's (1989) FrameChange Model, for which humor relies on a shift in frames, i.e. from a coherently apprehended one to a new one imposed on by the joke.

\subsection{Circle 2: Cultural frame}

People also store prototypical information about their culture and community. Instead of the personal storage of "I conceptualize X as $p$ " or "I believe that $p$ " that is typical of make-sense frames, in this case the individual acknowledges that certain information is typically cultural, stored as stereotypical chunks of information with the structure "in this culture, $\mathrm{X}$ is conceptualized as $p$ " or "in this culture it is believed that $p$." Both types of frames overlap to a certain extent, since some stereotypes may also be part of the individual's own beliefs, whereas other stereotypes are clearly not supported by the individual.

In previous research, I distinguished between the mental representations which people consider their own acquired thoughts (labeled private beliefs), and those which they regard as belonging to a community (labeled metarepresented cultural beliefs), and which may match, contradict, overlap with, or complement each other (cf. Yus 2002, 2004, 2005). The ability to have parallel representations of the same referent, one regarded as private and the other ascribed to a culture, is an interesting human capacity which improves interaction with the environment. In this sense, cultural stereotypes are normally salient to a whole community, thus becoming what is usually called collective representations attributed to a whole social group (often in the form of stereotypes), and they need not be erased when a person privately constructs parallel but differing beliefs about the same referent.

Needless to say, these cultural stereotypes are often the only source of humor in certain jokes and their humorous effects seem to lie in the acknowledgment or strengthening (and occasionally contradiction) of these stereotypes. 


\subsection{Circle 3: Utterance interpretation}

As was commented upon above, all the inferential steps leading to an interpretation of the utterance(s) in the joke may be exploited for the sake of humor, ranging from the identification of the logical form of the utterance to its inferential enrichment into a meaningful proposition, and also the construction of explicit and/or implicated interpretations (explicature/implicature). Needless to say, all jokes involve utterance interpretation and hearers have to fill the gap between what is coded in the joke and what is intended. The "joke interpretation circle" would be activated only when the inferential steps leading to an interpretation of the joke are manipulated by the speaker for the sake of humor. An example would be to play with disambiguation, i.e. when one sense of the word is clearly more likely to be selected but a second, more unlikely sense turns out to be the correct one.

\subsection{A new taxonomy of types of jokes}

These three sources of humor make up the ICM of humorous communication, which yields a taxonomy of seven types of jokes depending on whether the humorous strategy is based only on make-sense frames, cultural frames or utterance interpretation, or the speaker prefers to devise a strategy based on combinations of the Circles (these often collaborate to trick the hearer into selecting a certain interpretive path, see below). Seven types of jokes result depending on the activation of: (1) make-sense frame, cultural frame and utterance interpretation; (2) make-sense frame and cultural frame; (3) make-sense frame and utterance interpretation; (4) make-sense frame only; (5) cultural frame and utterance interpretation; (6) cultural frame only; and (7) utterance interpretation only.

This typology of jokes will be described and exemplified in the next Section, together with the results of the analysis of a large corpus of jokes.

\section{Analyzing jokes with the Intersecting Circles Model (ICM)}

\subsection{Method}

A corpus of 1000 jokes was randomly collected and analyzed according to this Model: 500 from the joke repository jokes2go (http://www.jokes2go.net/) and 500 from The Adult Joke Book (Sharpe 2004). Both sources comprise jokes in different sections containing commonly exploited sources of humor (jokes on bars, blondes, 
lawyers, doctors, religions, sex roles, etc.). These jokes were then analyzed and the underlying humorous strategies isolated. Next, the jokes were ascribed to any of the seven types predicted by the Model according to the shape of these strategies. In this sense, it should be stressed that, although the Model relies on three basic categories, these can only be said to be activated insofar as they do play a role in the generation of humorous effects. For example, hearers always make sense of the utterances they interpret and apply default frames to save mental effort. This is also at work in the case of jokes, but sometimes these frames are activated without really playing any role in the production of humorous effects, which lie, rather, in the other Circles, such as cultural stereotypes or utterance interpretation. Similarly, hearers always engage in an inferential enrichment of the words of the joke into a meaningful and contextualized proposition, since this is how interpretation of utterances invariably proceeds, but only when the inferential steps leading to an interpretation of the joke are manipulated for the sake of humor, would this joke be ascribed to the "utterance-interpretation Circle."

Finally, a second analysis was carried out in order to determine whether there are sub-patterns of humorous strategies inside the preliminary typology of the Model into seven categories.

\subsection{General analysis}

One of the objectives of this paper was to check how the jokes in the corpus fit into one of the seven categories in the ICM. Specifically, the jokes in which make-sense frames play a role amount to 701. Cultural frames were found in only 130 jokes. Finally the steps involved in utterance interpretation are manipulated for the sake of humor in 565 jokes.

\begin{tabular}{|c|c|c|c|c|}
\hline \multirow{2}{*}{$\begin{array}{l}\text { Global results } \\
\text { (701 jokes) }\end{array}$} & Type 1 & Type 2 & Type 3 & Type 4 \\
\hline & $13(1.3 \%)$ & $22(2.2 \%)$ & $317(31.7 \%)$ & $\begin{array}{c}349 \\
(34.9 \%)\end{array}$ \\
\hline $\begin{array}{c}\text { Type } 3 \\
\text { [make-sense / } \\
\text { utterance } \\
\text { interpretation] } \\
\text { (317 jokes) }\end{array}$ & \multicolumn{4}{|c|}{$\begin{array}{l}\text { Making sense of the joke with implicated premises/conclusions } \\
(165,52.05 \%) \\
\text { Two valid interpretations of some portion of text }(124,39.11 \%) \\
\text { a. Ambiguity at the beginning of utterance }(4,1.26 \%) \\
\text { b. Ambiguity in the middle of utterance }(78,24.60 \%) \\
\text { c. Ambiguity at the end of utterance }(42,13.25 \%) \\
\text { Two interpretations of the whole joke possible, only one valid } \\
\text { (21, } 6.62 \%) \\
\text { No resolution of incongruity, simultaneous entertainment }(7,2.20 \%)\end{array}$} \\
\hline
\end{tabular}




\begin{tabular}{|c|l|}
\hline Type 4 & Mild incongruity-resolution pattern $(182,52.14 \%)$ \\
$\begin{array}{c}\text { [make-sense] } \\
\text { (349 jokes) }\end{array}$ & $\begin{array}{l}\text { Two interpretations of whole joke possible, only one valid }(23,6.59 \%) \\
\text { No resolution of incongruity, simultaneous entertainment }(4,1.14 \%)\end{array}$ \\
\hline
\end{tabular}

Table 1. Make-sense frame results.

Table 1 shows the results of the analysis of jokes in which make-sense frames play a part in the generation of humorous effects (types 1,2, 3 and 4), and results for type 3 (make-sense frame and utterance interpretation) and type 4 (make-sense frame) are analyzed in more detail. Table 2 shows the sub-categories of jokes in which some form of social/cultural stereotype is strengthened or contradicted (types 1, 2, 5 and 6). Finally, Table 3 lists the percentages of jokes in which utterance interpretation is manipulated for the sake of humor (types 1, 3, 5 and 7), with special emphasis on type 3 (make-sense frame, utterance interpretation) and type 7 (utterance interpretation).

\begin{tabular}{|c|c|c|c|c|}
\hline $\begin{array}{c}\text { Global results } \\
(130 \text { jokes })\end{array}$ & Type 1 & Type 2 & Type 5 & Type 6 \\
\cline { 2 - 5 } & $13(10 \%)$ & $22(16.92 \%)$ & $31(23.84 \%)$ & $64(49.23 \%)$ \\
\hline
\end{tabular}

Table 2. Cultural frame results.

\begin{tabular}{|c|c|c|c|c|}
\hline \multirow{2}{*}{$\begin{array}{l}\text { Global results } \\
\text { (565 jokes) }\end{array}$} & Type 1 & Type 3 & Type 5 & Type 7 \\
\hline & $13(2.30 \%)$ & $317(56.10 \%)$ & $31(5.48 \%)$ & $204(36.10 \%)$ \\
\hline $\begin{array}{c}\text { Global results } \\
\text { [inferential strategies } \\
\text { to reach an } \\
\text { interpretation of the } \\
\text { joke exploited for the } \\
\text { sake of humor] }\end{array}$ & \multicolumn{4}{|c|}{$\begin{array}{l}\text { Extraction of logical form from coded words }(7,1.41 \%) \\
\text { Reference assignment }(63,11.5 \%) \\
\text { Disambiguation }(177,30.74 \%) \\
\quad \text { a. polysemy }(126,22.30 \%) \\
\quad \text { b. homophony }(8,1.41 \%) \\
\quad \text { c. paronymy (phonetic similarity) }(37,6.54 \%) \\
\text { d. sub-sentential utterances }(6,1.05 \%) \\
\text { Free enrichment }(12,2.12 \%) \\
\text { Concept adjustment }(123,21.76 \%) \\
\text { Playing with explicit/implicit interpretations }(35,6.18 \%) \\
\text { Retrieval/derivation of implicated premises and conclusions } \\
(217,38.40 \%)\end{array}$} \\
\hline
\end{tabular}




\begin{tabular}{|c|c|}
\hline $\begin{array}{c}\text { Type } 3 \\
\text { [make-sense / } \\
\text { utterance } \\
\text { interpretation] } \\
\text { (317 jokes) }\end{array}$ & $\begin{array}{l}\text { Extraction of logical form from coded words }(2,0.65 \%) \\
\text { Reference assignment }(27,8.51 \%) \\
\text { Disambiguation }(88,27.76 \%) \\
\quad \text { a. polysemy }(78,24.60 \%) \\
\text { a.1. make-sense frame facilitates choice of one sense ( } \\
\quad 63,19.87 \%) \\
\text { a.2. make-sense frame simply activated }(15,4.73 \%) \\
\text { b. homophony }(3,0.94 \%) \\
\text { c. paronymy (phonetic similarity) }(7,2.20 \%) \\
\text { Free enrichment }(4,1.26 \%) \\
\text { Concept adjustment }(44,13.88 \%) \\
\text { Playing with explicit/implicit interpretations }(8,2.52 \%) \\
\text { Retrieval/derivation of implicated premises and conclusions } \\
(184,58.04 \%)\end{array}$ \\
\hline $\begin{array}{c}\text { Type } 7 \\
\text { [utterance } \\
\text { interpretation] } \\
\text { (204 jokes) }\end{array}$ & $\begin{array}{l}\text { Extraction of logical form from coded words }(5,2.45 \%) \\
\text { Reference assignment }(28,13.72 \%) \\
\text { Disambiguation }(82,40.19 \%) \\
\quad \text { a. polysemy }(44,21.56 \%) \\
\quad \text { b. homophony }(5,2.45 \%) \\
\text { c. paronymy (phonetic similarity) }(28,13.72 \%) \\
\text { d. sub-sentential utterance }(5,2.45 \%) \\
\text { Free enrichment }(6,2.94 \%) \\
\text { Concept adjustment }(67,32.84 \%) \\
\text { Playing with explicit/implicit interpretations }(26,12.74 \%) \\
\quad \text { a. implicit interpretation tuned explicit }(22,10.78 \%) \\
\text { b. explicit interpretation turned implicit }(4,1.96 \%) \\
\text { Retrieval/derivation of implicated premises and conclusions } \\
(15,7.35 \%)\end{array}$ \\
\hline
\end{tabular}

Table 3. Utterance interpretation results.

\subsection{Specific types of jokes in the corpus}

Joke type 1: Make-sense frame, cultural frame, utterance interpretation. In this joke type, the speaker's humorous strategy involves acting upon the hearer's utterance interpretation, the activation of make-sense frames and the recognition of cultural frames. Not many instances in the corpus fit this triple intersection of Circles (only 13 of them), but those which do reveal a very interesting humorous strategy. Consider the joke in (5) (Attardo 2008: 1205):

(5) The pilot of a plane flying over the ocean comes on the intercom and announces that both engines are malfunctioning and the plane will crash in twenty minutes. Panic ensues. A young woman stands up and says: 'I have never been with a man. I want to 
experience that before I die. Is there anyone who will make me feel like a real woman?' A young man stands up, rips off his shirt, hands it to her and says: 'Here! Iron this!'

In (5), the hearer proceeds with the interpretation following a relevance-seeking criterion and makes sense of the airplane situation and the fact that there is an upcoming disaster facilitates the choice of "make love with a man" as the most relevant interpretation of "be with a man," the coded concept of the word "man" being conceptually adjusted (narrowed) to meet the expectations of relevance (not any man but "a man who is a lover"). The same applies to "make me feel like a real woman," which is easily interpreted as "give me pleasure" within the initiated sexual frame (while "woman" is adjusted into "a person given full sexual pleasure"). The end of the joke (Iron this!) brings incongruity into the interpretation and forces the hearer to engage in inferential backtracking and reinterpreting the sexual phrase differently (feel like a real woman by performing a stereotypical female activity). Both the phrase and the sexual make-sense frame are potentially valid but the hearer had not noticed the latent possibilities because these are not as relevant. At the same time, the cultural stereotype of "woman as housewife" is conveyed and strengthened, providing additional relevance and justification for the increased mental effort.

Joke type 2: Make-sense frame, cultural frame. In this type of joke, make-sense frames and cultural frames are activated in the search for humorous effects. 22 instances were isolated in the corpus. For example, joke (6), in which the hearer makes sense of the scenario of the joke and concludes that women are inferior to men (which in parallel corroborates the pervasive sex-role stereotype found in jokes), only to be invalidated at the end. The humor lies in contradicting the justactivated cultural frame as a consequence of the activation of an accessible makesense frame.

(6) A man went to the doctor's. The doctor came in and said, "Well, I've got some good news and some bad news. The bad news is that you have an inoperable brain tumor. The good news is our hospital has just been certified to do brain transplants. The man's brain costs $\$ 100,000$ and the woman's brain costs $\$ 30,000$." The patient could not help but ask, "Why such a large difference between the male and the female brain?" The doctor replied, "The female brain is used."

Joke type 3: Make-sense frame, utterance interpretation. Roughly one third of the jokes fit this type (317; see Table 1 and Table 3 for a detailed analysis of these type-3 jokes in the corpus). In this kind of joke, the inferential steps leading to an interpretation are manipulated by the speaker, who also predicts the construction of a make-sense frame. This frame plays a central role in directing the hearer towards the choice of an apparently relevant interpretation in its balance of interest (cognitive effects) and mental effort, which is invalidated. 
Several patterns in the combination of make-sense frames and utterance interpretation arise from the corpus (cf. Table 1). Firstly, make-sense frames often entail the retrieval of implicated premises and the derivation of implicated conclusions (165 jokes, 52.05\%). These implications are, at the same time, part of the interpretation of the utterance, triggered by the hearer's search for a relevant interpretation of the joke. Therefore, a blurred intersecting area is created in which implications are needed both for building up a make-sense frame and for reaching an appropriate interpretation of the utterance. For instance, the humorous effects of joke (7a) below lie in the hearer's retrieval of the implicated premise (7b) and derivation of the implicated conclusions (7c) about the effects of marriage, which can be considered both part of utterance interpretation and part of the hearer's construction of a make-sense frame for the end of the joke:

(7) a. A dietician was once addressing a large audience in Chicago. "Red meat is awful. Vegetables can be disastrous, and none of us realizes the germs in our drinking water. But there is one thing that is the most dangerous of all and we all of us eat it. Can anyone here tell me what lethal product I'm referring to? You, sir, in the first row, please give us your idea." The man lowered his head and said, "Wedding cake."

b. Implicated premise: Wedding cake is served after marriage ceremonies.

c. Implicated conclusions: He thinks marriage is a bad idea; he has suffered from a bad experience with marriage. ${ }^{3}$

Secondly, make-sense frames also combine with some potentially ambiguous part of the joke to generate humorous effects, for example by making one sense of a polysemous word more relevant and easy to select. Although I predicted this ambiguous part to be mostly located at the end of the joke and fit the incongruityresolution pattern (Suls 1972), more instances were found to fit the setupincongruity-resolution (or SIR) pattern (Attardo 1997), with the ambiguous chunk of text located roughly in the middle of the joke (78 jokes, $24.60 \%)$. This fits nicely with the "incremental" relevance-seeking interpretive procedure in which the assumptions from previously processed discourse form a preliminary background for the interpretation of subsequent chunks of text, as happens with the disambiguation of "bat" in the following example, facilitated by the preceding text:

(8) a. Your team is disqualified from the baseball game. Peter's bat is too grey.

b. We've chosen John's mouse for the experiment. Peter's bat is too grey.

In the case of many type- 3 jokes, the hearer first builds up a make-sense scenario that saves effort for the interpretation of subsequent stretches of discourse. The choice of an interpretation of the ambiguous part is facilitated by the assumptions still active from previously processed discourse and by expectations of frame congruence, only to be invalidated at a subsequent part of the joke. 
Finally, a resolution is found in a third phase, normally resolved by the hearer's inferential backtracking to re-interpret the joke, as in (9) and (10):

(9) A policeman stops a lady and asks for her license. He says "Lady, it says here that you should be wearing glasses." The woman answered "Well, I have contacts." The policeman replied "I don't care who you know! You're getting a ticket!"

(10) A doctor and a nurse were called to the scene of an accident. Doctor: We need to get these people to a hospital now! Nurse: What is it? Doctor: It's a big building with a lot of doctors, but that's not important now!

In these jokes, the make-sense frame in the setup makes it easy for the hearer to find a relevant (i.e. accessible) meaning for "contacts" (without realizing that it is polysemous) and a valid referent for "it" in "What is it?" both located in the middle of the joke. The next part of the joke creates the incongruity, provoking an inferential backtracking. This is costly in terms of mental effort but compensated for by an offset of cognitive (i.e. humorous) effects.

A third -and less frequent- pattern found in the corpus is when two interpretations of the whole utterance (not of part of it) are possible, only one of which is eventually correct (21 jokes, 6.62\%). In (11), for example, at the end of the joke we are reminded that the whole joke has to be disambiguated and reinterpreted:

(11) It was laying limp in my hand. It was very long, kind of thin. I slid it between my fingers until I got to the end of it. I was turning it on. It became firm in my hands, and the end was wet. Then it got very hard and began gushing out of the tip. Then I took the garden hose and watered the bushes.

Finally, in a fourth pattern the incongruity is left unresolved, since both interpretations are valid and the hearer is invited to process and entertain them in parallel (7 instances, 2.20\%). This pattern fits Ritchie's (2009: 6) alternative interpretation contrast, according to which neither interpretation is defined as having priority, with the incongruity arising from the mutual clash, as happens in (12):

(12) A young husband with an inferiority complex insisted he was just a little pebble on a vast beach. The marriage counselor, trying to be creative, told him, "If you wish to save your marriage, you'd better be a little boulder."

As in any metaphor, this joke entails a conceptual adjustment of "pebble" so that the underlying concept can be mapped onto a person. The end of the joke entails a disambiguation of "boulder" (paronymy, phonetic similarity) into "boulder/bolder" and generates two interpretations, both of which are valid and entertained simultaneously without the hearer being able to choose the most 
relevant one. The humorous effects lie precisely in the entertainment of these simultaneous possibilities.

The analysis of sub-strategies for utterance interpretation in joke type 3 (cf. Table 3) revealed that, besides ambiguous terms, and as predicted in previous research (Yus 2003, 2008, 2012a), all the stages in utterance interpretation can be exploited for the sake of humor, ranging from the initial decoding of the words uttered into a logical form (as in (13) below) to the inferential comprehension of explicit and implicated meanings with the aid of context. The most frequent strategy is the aforementioned retrieval/derivation of implicated premises and conclusions in parallel to building up make-sense frames. Disambiguation, already mentioned above, is also frequent $(27.76 \%)$, with polysemy as the most common sub-task (24.60\%), also at work for "field" in joke (13) below. Concept adjustment is exploited in several jokes which play with expected vs. unexpected ad hoc meanings of coded concepts in context (13.88\%).

(13) A man is driving down a country road, when he spots a farmer standing in the middle of a huge field of grass. He pulls the car over to the side of the road and notices that the farmer is just standing there, doing nothing, looking at nothing. The man gets out of the car, walks all the way out to the farmer and asks him, "Ah excuse me mister, but what are you doing?" The farmer replies, "I' $m$ trying to win a Nobel Prize." "How?" asks the man, puzzled." Well, I heard they give the Nobel Prize... to people who are out standing in their field."

Logical form 1: people who are [out] [standing] [in their field].

Logical form 2: people who are [outstanding] [in their field].

6.3.4. Joke type 4: Make-sense frame. 349 jokes resort to make-sense frames as the main or only source of humorous effects, without needing the aid of the manipulation of utterance interpretation or the activation of cultural frames. All of the jokes in this type comply with the incongruity-resolution pattern (cf. Table 1). However, $40.11 \%$ of these type-four jokes offer a very mild form of incongruity that generates little cognitive dissonance. This sub-type contrasts with a more surprising incongruity-resolution pattern à la Suls (1972) in 52.14\% of the jokes. These are exemplified in jokes (14) and (15) respectively:

(14) Sherlock Holmes and Doctor Watson went on a camping trip to Dartmoor and as they lay down for the night Sherlock Holmes said, "Doctor Watson, my old friend, when you look up into the darkness, please tell me what you see." "Well, I can see a very clear sky, there are no clouds... the Milky Way and the planet Venus." Watson laughed and said, "But knowing you, Sherlock, I'm sure there are many things I have missed. What have you deduced?" There was a moment's silence and then Holmes replied, "Somebody's nicked our tent."

(15) Bill sat alone in the hospital room at his dying wife's beside. Her voice was little 
more than a hoarse whisper. "Bill darling," she breathed. "I've got a confession to make before I go... I ... I'm the one who took the $\$ 10,000$ from your safe in the house... I spent it on a fling with your best friend Jimmy..." "That's all right dearest; don't even give it a second thought." said Bill. "I have a small confession too. I'm the one who poisoned you."

6.3.5. Joke type 5: Cultural frame, utterance interpretation. Sometimes cultural frames and utterance interpretation are jointly exploited for the humorous outcome of the joke. Only 31 instances of the corpus fit this type. Most of them strengthen a cultural stereotype with the aid of disambiguation, as in (16), in which the humorous outcome is based on strengthening the negative qualities of lawyers (cultural frame) but also on the resolution of the ambiguity of "serve" (utterance interpretation):

(16) A man and his alligator walk into a bar and the man asks, "Does this bar serve lawyers?" "Of course we do," replied the bartender." Great," said the man, "I'd like a beer... and give me a lawyer for my alligator."

6.3.6. Joke type 6: Cultural frame. For 64 jokes in the corpus, strengthening or contradicting cultural frames seems to be the only source of humor, with no need of either make-sense frames or manipulated steps for utterance interpretation (cf. Yus 2002, 2004, 2005). Stereotypes include professions, sex roles, national identities, specific connoted places, family roles, etc. An example of one of these stereotypes is provided in (17):

(17) Q: When does a person decide to become an accountant?

A: When he realizes he doesn't have the charisma to succeed as an undertaker.

6.3.7. Joke type 7: Utterance interpretation. Finally, as analyzed in previous research (Yus 2003, 2008, 2012a, forthcoming), some manipulation of the interpretive strategies leading to a relevant interpretation of the joke seem to be the only source of humorous effects in 204 jokes. These typically play with polysemy, ambiguity, punning, conceptual adjustment, the explicit/implicit likelihood of interpretations and the derivation of implications with the aid of context. Table 3 lists the specific relevance-seeking inferential strategies and percentages (for the jokes in the corpus) which are manipulated for the sake of humor. In this joke type, after the hearer has identified the logical form of the stretches of text, the speaker can predict that he/she will engage in its inferential enrichment into a meaningful contextualized interpretation. All the sub-strategies involved in this inferential activity can be exploited for the generation of humorous effects, starting from reference assignment (28 jokes, $13.72 \%$ ). Concerning disambiguation, polysemy is the most typical source of humor (44 jokes, $21.56 \%$ ), above paronymy ( 28 jokes, 
$13.72 \%$ ), homophony (5 jokes, $2.45 \%$ ) and ambiguous sub-sentential utterances (5 jokes, $2.45 \%$ ). Free enrichment was not frequently used as a source of humor (6 jokes, 2.94\%). By contrast, concept adjustment was relatively often used as a source of humorous effects. (67 jokes, 32.84\%), especially those containing metaphors, since all of them involve conceptual adjustment, as happens with "woman is an ice cube" in (18):

(18) A man went into a bar and ordered a gin and tonic. When it was placed before him, he exclaimed, "My goodness, an ice cube with a hole in it, that's new." "No it isn't," commented a sullen looking man sitting next to him. "I married one."

The outcome of these inferential strategies is the explicit content of the joke, now fully contextualized and communicated as an explicature. This interpretation can then be used as one of the premises for the derivation of implicatures. In this sense, 26 jokes $(12.74 \%)$ fitting joke-type 7 play with alternative explicit versus implicit meanings of phrases. The humorous pattern is to force the hearer into entertaining simultaneously both the accessible and typical implicit interpretation of a phrase (e.g. an idiom) and its literal counterpart, as in (19):

(19) The phone rings and the husband answers it. "No, mate, you want the Met Office." "Who was that, darling?" asks the wife. "I don't know, I think he wanted the weather forecast, because he asked me if the coast was clear."

\section{Concluding remarks and prospects of research}

The ICM predicts that all jokes involve the manipulation of any of three areas (Circles) or their combinations: make-sense frame, cultural frame and utterance interpretation, aided by the prediction of the hearer's relevance-seeking inferential steps. The analysis of a corpus of 1000 jokes corroborated this prediction. However, the corpus is mainly made up of jokes in the Anglosaxon context, since the repository jokes2go.net contains American jokes and The adult joke book is a selection of English jokes. It would be interesting to check the extent to which jokes from a different cultural context yield the same percentages and subcategories as the ones commented upon in this paper. Many of the jokes analyzed for this paper will probably suit any cultural context, whereas others will be more typical in a specific cultural area. Besides, it would be interesting to check the influence of the languages themselves in the frequency of specific joke types. Speakers may take advantage of the formal features and resources of languages differently in order to create humorous effects. Hence the percentage of jokes involving a manipulation of (decoding and) inferential steps associated with their phonotactic constraints, homonymy, polysemy, phrasal boundaries, intra-sentential 
structures, etc. may be higher in some languages than in others. This will be addressed in future research.

\section{Notes}

1. I would like to thank Luz Gil, José Mateo, Manuel Padilla and Agnieszka Piskorska for their comments on a draft of this paper.

2. Utterance interpretation will be used in italics when referring to one of the Circles in the ICM.

3. A reviewer of this paper pointed out that joke (7) also involves the activation of the "cultural frame circle." In the context of this paper, cultural frames involve information about cultures, countries, professions, etc. that the hearer regards as stereotypical, as part of the collective storage of information (in this culture it is believed that $p$ ), and often not matching his/her personal beliefs and opinions on the same referent (I believe that $p$ ). In my opinion, there is no cultural stereotype of the type "marriage is harmful for one's health," but if that was the case, then joke (7) would shift to Type 1.

\section{References}

Attardo, Salvatore. 1997. The semantic foundations of cognitive theories of humor. Humor 10(4). 395-420.

Attardo, Salvatore. 2008. Semantics and pragmatics of humor. Language and Linguistics Compass 2(6). 1203-1215.

Attardo, Salvatore. 2011. Humor. In Jan Zienkowski, Jan-Ola Östman \& Jef Verschueren (eds.), Discursive Pragmatics, 135-155. Amsterdam \& Philadelphia: John Benjamins.

Attardo, Salvatore \& Victor Raskin. 1991. Script theory revisi(it)ed: Joke similarity and joke representation model. Humor 4. 293-347.

Bednarek, Monika A. 2005. Frames revisited-the coherence-inducing function of frames. Journal of Pragmatics 37. 685-705.

Biegajło, Magdalena. 2012. A relevance-theoretic classification of jokes: A critical survey. Paper presented at Interpreting for Relevance: Discourse and Translation, University of Warsaw, 25-26 September.

Coulson, Seana. 2001. Semantic Leaps. Frame-Shifting and Conceptual Blending in Meaning Construction. Cambridge: Cambridge University Press.

Dynel, Marta. 2010. How do puns bear relevance? In Marta Kisielewska-Krysiuk, Agnieszka Piskorska \& Ewa Walaszewska (eds.), Relevance studies in Poland. Exploring translation and communication problems, 105-124. Warsaw: 
Warsaw University Press.

Dynel, Marta. 2012a. Garden paths, red lights and crossroads: On finding our way to understanding the cognitive mechanisms underlying jokes. Israeli Journal of Humor Research 1(1). 6-28.

Dynel, Marta. 2012b. What RT cannot do, IR can: On the incremental interpretation of jokes in (non)relevance-theoretic terms. In Ewa Walaszewska \& Agnieszka Piskorska (eds.), Relevance theory. More than understanding, 147-166. New Castle upon Tyne: Cambridge Scholars Publishing.

Giora, Rachel. 1991. On the cognitive aspects of the joke. Journal of Pragmatics 16, 465-485.

Koestler, Arthur. 1964. The Act of Creation. New York: MacMillan.

Raskin, Victor. 1985. Semantic Mechanisms of Humor. Dordrecht: Kluwer Academic.

Ritchie, Graeme. 2004. The Linguistic Analysis of Jokes. London: Routledge.

Ritchie, Graeme. 2006. Reinterpretation and viewpoints. Humor 19(3). 251-270.

Ritchie, Graeme. 2009. Variants of incongruity-resolution. Journal of Literary Theory 3(2). 1-20.

Scollon, Ron \& Suzanne Wong Scollon. 2001. Intercultural communication. A discourse approach. Oxford: Blackwell.

Sharpe, Johnny. 2004. The Adult Joke Book. London: Capella.

Sperber, Dan \& Deirdre Wilson. 1995. Relevance. Communication and Cognition (second edition). Oxford: Blackwell.

Suls, Jerry M. 1972. A two-stage model for the appreciation of jokes and cartoons: An information-processing analysis. In Jeffrey H. Goldstein \& Paul E. McGhee (eds.) The Psychology of Humor. Theoretical Perspectives and Empirical Issues, 81-100. New York: Academic Press.

Tannen, Deborah. 1993. What's in a frame? Surface evidence for underlying expectations. In Deborah Tannen (ed.), Framing in discourse, 403-418. Oxford: Oxford University Press.

Wenzel, Peter. 1989. Von der Struktur des Witzes zum Witz ser Struktur: Studies zur Technik der Pointierung. Heidelberg: Winter.

Wilson, Deirdre. 1994. Relevance and understanding. In: Gillian Brown, Kirsten Malmkjaer, Alastair Pollitt \& John Williams (eds.), Language and Understanding, 35-58. Oxford: Oxford University Press.

Wilson, Deirdre \& Dan Sperber. 2002. Relevance theory. UCL Working Papers in Linguistics 14. 249-290.

Yus, Francisco. 1997. La teoría de la relevancia y la estrategia humorística de la incongruencia- resolución. Pragmalingüística 3(4). 497-508.

Yus, Francisco. 2002. Stand-up comedy and cultural spread: The case of sex roles. Babel A.F.I.A.L., special issue on humor studies. 245-292. 
Yus, Francisco. 2003. Humor and the search for relevance. Journal of Pragmatics 35(9). 1295-1331.

Yus, Francisco. 2004. Pragmatics of humorous strategies in El club de la comedia. In Rosina Márquez-Reiter \& María Elena Placencia (eds.), Current trends in the pragmatics of Spanish, 320-344. Amsterdam \& Philadelphia: John Benjamins.

Yus, Francisco. 2005. Dave Allen's stand-up monologues: An epidemiological approach. In José Mateo \& Francisco Yus (eds.), Thistles. A homage to Brian Hughes (volume 2: Essays in Memoriam), 317-344. Alicante: University of Alicante, Department of English Studies.

Yus, Francisco. 2008. A relevance-theoretic classification of jokes. Lodz Papers in Pragmatics 4(1). 131-157.

Yus, Francisco. 2012a. Strategies and effects in humorous discourse. The case of jokes. In Bárbara Eizaga Rebollar (ed.), Studies in Linguistics and Cognition, 271-296. Berlin: Peter Lang.

Yus, Francisco. 2012b. Relevance, humour and translation. In Ewa Walaszewska \& Agnieszka Piskorska (eds.), Relevance theory. More than understanding, 117-145. New Castle upon Tyne: Cambridge Scholars Publishing.

Yus, Francisco. forthcoming. An inference-centered analysis of jokes: The Intersecting Circles Model of humorous communication. In Leonor Ruiz Gurillo \& Belén Alvarado (eds.), Irony and humor: Highlights and genres. Amsterdam \& Philadelphia: John Benjamins. 